\title{
REVISTA PERUANA DE MEDICINA EXPERIMENTAL Y SALUD PÚBLICA: GENERANDO UNA CULTURA DE PUBLICACIÓN EN EL PERÚ
}

\author{
REVISTA PERUANA DE MEDICINA EXPERIMENTAL Y SALUD PÚBLICA: GENERATING A \\ PUBLICATION CULTURE IN PERU
}

César Cabezas ${ }^{1, a}$

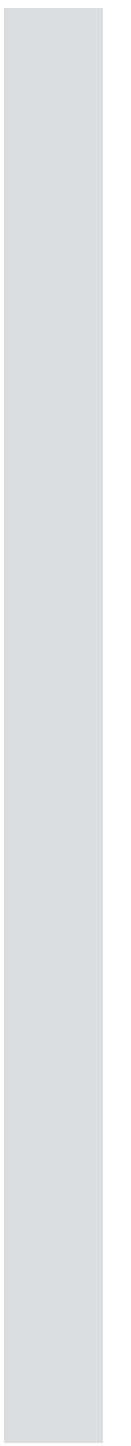

Para enfrentar los retos en salud y viabilizar la transformación hacia un sistema de salud con equidad y eficiencia, es necesario generar una cultura de investigación responsable y ética, priorizada en temas de interés nacional (1), junto a ello fomentar y continuar la publicación de estas investigaciones, lo que permitirá que los tomadores de decisiones cuenten con información oportuna y de calidad.

En el Perú, durante el periodo 2000-2009, si bien se ha mostrado un incremento de publicaciones científicas (2), nuestra producción científica para el año 2009 es menor que la de países que tienen una mayor asignación de recursos del PBI para investigación y desarrollo, como Argentina (6197 publicaciones); Brasil (26 482 publicaciones); Chile (3646 publicaciones), y México (8262 publicaciones) ${ }^{(3)}$. Sin embargo, no todo está relacionado solamente con el financiamiento, sino también al cómo nos organizamos y desarrollamos investigación y difundimos resultados, en este contexto y sin dejar de transigir por más recursos, y en ese tenemos tímidamente aún dos revistas en el país que se encuentran en sistemas científicos de difusión mundial, siendo la nuestra, la única editada por una institución pública.

En general, la producción científica peruana es todavía escasa, con un alto porcentaje de colaboración extranjera ${ }^{(2)}$, que años anteriores se realizaban en atención de problemas de salud no necesariamente de interés nacional. Además, la investigación en salud se centralizaba en pocos sectores, con una casi nula participación de instituciones del interior del país. Pero esto no se debía necesariamente a la poca inversión en investigación, o a que no se disponían de centros o institutos de investigación, sino a la ausencia de una cultura de publicación.

La cultura de publicación consiste en: identificar un problema, estudiarlo y hacer de conocimiento público la nueva información a través de su publicación, lo que permitirá su posterior aplicación. En el contexto peruano, muchas instituciones se quedan en el estudio del problema e intentos de solución, sin comunicar dichas experiencias que podrían ser exitosas al aplicarlas a otras realidades. Entonces, la falta de publicación afectaba negativamente la toma de decisiones en salud, dado que los gestores o quienes determinaban las políticas en salud, no disponían de suficiente información para sustentar las medidas a adoptar, empleando experiencias limitadas, de contextos no extrapolables al Perú o sin un adecuado análisis.

Estas medidas no sustentadas significarían un gasto inadecuado de los recursos del Estado. Ante ello, la Revista Peruana de Medicina Experimental y Salud Pública (RPMESP) ha implementado diversas estrategias para brindar a la comunidad en general, información científicamente válida para una mejor toma de decisiones, participando de forma activa en la generación de una cultura de publicación responsable, llenando un vacío al facilitar el acercamiento de la comunidad científica-académica con los profesionales de la salud y los decisores políticos; brindando información oportuna, práctica, gratuita y de calidad, de publicaciones científicas de primer nivel generadas en el país y la región, para un mejor quehacer en salud y para la toma de decisiones en políticas de salud en beneficio de la población.

Instituto Nacional de Salud. Lima, Perú.

Editor general de la RPMESP, médico infectólogo-tropicalista

Recibido: 02-08-12 Aprobado: 08-08-12

Citar como: Cabezas C. Revista Peruana de Medicina Experimental y Salud Pública: Generando una cultura de publicación en el Perú. Rev Peru Med Exp Salud Publica. 2012;29(3):301-2. 
Para efectivizar la difusión y uso de estas publicaciones entre los profesionales de la salud y los decisores políticos, la RPMESP ha implementado estrategias de innovación y optimización, como: a) acceso libre y a texto completo a la información vía Internet; a través de diversos sistemas de búsqueda comunes (Google) o especializados (MEDLINE, SCOPUS, EMBASE); b) distribución gratuita de la revista impresa entre investigadores, docentes universitarios, políticos, decisores en salud, etc.; c) convocatoria a especialistas nacionales y extranjeros quienes brindan enfoques alternativos para enfrentar nuestros problemas de salud pública; d) publicación de trabajos sobre temas de relevancia nacional en salud, ejemplo de ello son los números dedicados al desarrollo de recursos humanos, acceso universal a la salud, violencia y adicciones, nutrición, etc.; e) implementación de un sistema informático de procesos editoriales on-line; f) alertas informativas a través de suscripciones gratuitas en nuestra página web; y g) interacción con la población a través de redes sociales.

Todo ello se complementa con nuestras actividades docentes dirigidas a investigadores, profesionales y alumnos de ciencias de la salud, a través de capacitaciones descentralizadas en temas de importancia para la investigación, motivándolos a que realicen investigaciones en sus regiones para atender sus prioridades sanitarias y que terminen en publicaciones.

El impacto de la RPMESP se ve reflejado en tres niveles: 1) en nuestros procesos, al cumplir con certificaciones internacionales en publicación científica de alto nivel, ingresando a importantes bases de datos a nivel mundial; 2) brindando aportes para una mejor gestión de la salud, priorizando temas de interés nacional como: enfermedades transmisibles, evaluación económica de vacunas, en medicamentos, aseguramiento universal, etc; y 3) en la producción científica nacional, al incrementar en $20 \%$ la visualización de la actividad científica peruana a la producción mundial, sirviendo de base para la construcción de otras investigaciones.

Para lograr lo alcanzado, además de superar problemas de financiamiento, hemos necesitado de recursos humanos de manera permanente y comprometidos con las estrategias implementadas; en consecuencia de estos cambios, y con mucho esfuerzo y dedicación, la RPMESP ha contribuido a generar una cultura de investigación ética, responsable y comprometida con el desarrollo del Perú, lo cual ha merecido su reconocimiento como una de las Buenas Prácticas en Gestión Pública 2012, por la organización Ciudadanos al Día, la cual es una entidad privada sin fines de lucro, que busca contribuir con la reforma del Estado y el empoderamiento ciudadano, a través de la promoción de un Estado más transparente, eficiente e inclusivo, y una ciudadanía más informada, exigente y participativa.

Sin embargo, aún hay mucho por recorrer en este camino de fomentar y practicar una cultura de publicación en salud.

\section{REFERENCIAS BIBLIOGRÁFICAS}

1. Cabezas C.Necesidad delainvestigación en salud para contribuir a la equidad, la salud y el desarrollo. Rev Peru Med Exp Salud Publica. 2010;27(3):310-1.

2. Huamaní C, González AG, Curioso WH, Pacheco-Romero J. Redes de colaboración y producción científica sudamericana en medicina clínica, ISI Current Contents 2000-2009. Rev Med Chile. 2012;140(4):466-75.

3. Red de Indicadores de Ciencia y Tecnología (RICYT) [Internet].
Buenos Aires: RICYT; c2009 [citado el 2 de julio del 2012]. Disponible en: http://bd.ricyt.org/explorer.php/ query/submit? country $\% 5 \mathrm{~b} \% 5 \mathrm{~d}=\mathrm{PE} \&$ syear $=1990$ \&eyear $=2009$ 